\section{¿Es posible generalizar en estudios cualitativos?}

Is it possible to generalize in qualitative studies?

\section{José Luis Ventura-León ${ }^{1}$} Miguel Barboza-Palomino ${ }^{1}$

${ }^{1}$ Facultad de Ciencias de la Salud, Universidad Privada del Norte. Lima Perú. mbarbozapalomino@outlook.com.pe

Ciência \& Saúde Coletiva se caracteriza por la apertura a la publicación de estudios cualitativos y discusiones al respecto. En ese sentido, un reciente número fue nominado "Investigación cualitativa para pensar y actuar en el campo de la salud". No obstante, lo descrito aún constituye una realidad ajena a las revistas vinculadas al ámbito de la salud, en las que destaca la valoración preferente de investigaciones que siguen análisis estadísticos y muestreo aleatorizado ${ }^{1}$.

A pesar de, en los últimos años está aumentando la tendencia para la realización de estudios cualitativos, ya que, en el campo de la salud, donde intervienen múltiples factores, éstos permiten generar información valiosa acerca del contexto, experiencias y significados que construyen las personas ${ }^{2}$. Sin embargo, siguen vigentes argumentos tradicionales que cuestionan la utilidad del conocimiento que se construye, resaltando los que afirman la ausencia de generalizaciónde los resultados de investigaciones cualitativas ${ }^{3}$. Considerando lo mencionado, se plantea la pregunta: ¿Es posible generalizar estudios cualitativos? La presente misiva apunta a brindar algunas repuestas.

Al respecto, el principio de generalización,entendido como el grado en el cual los hallazgos de un estudio son aplicables a otras muestras o poblaciones, requiere interpretarse desde otra perspectiva que valore la naturaleza de la investigación cualitativa, cuyos resultados no buscan obtener leyes o principios, sino, examinar comprensivamente a los actores y contextos involucrados en el tema de estudio 4 .

Con relación a lo anteriormente expuesto, se reconoce la existencia de dos formas de generalización, una de tipo nomotética (vinculada a estudios cuantitativos) y otra de tipo ideográfica, ésta última relacionada con investigaciones cualitativas ${ }^{5}$, pudiendo afirmarse, a partir de ello, que es posible generalizar los resultados de estudios cualitativos.Incluso, hace varios años, existen términos propuestos por teóricos y metodólogos cualitativos, tal es el caso de transferibilidad, entendido como la posibilidad de extender los hallazgos a otras poblaciones ${ }^{6}$. No obstante, la aplicación de tal principio amerita considerar diferentes aspectos, básicamente de similitud, en relación a las características del lugar, los participantes del estudio y el tema abordado ${ }^{5}$. Así también, resulta importante garantizar la calidad de las investigaciones cualitativas.

Se concluye que es factible generalizar los hallazgos de estudios cualitativos que cumplan con avalar la calidad metodológica en su ejecución. Lo descrito, invita a investigadores escépticos con la investigación cualitativa a cuestionar su perspectiva en relación a la utilidad e impacto del conocimiento que se puede generar. De igual forma, contribuye a la reflexión de investigadores que desarrollan estudios cualitativos en garantizar su calidad. Finalmente, permite afirmar la validez de los hallazgos de estudios cualitativos para la toma de decisiones en salud ${ }^{7}$.

\section{Referencias}

1. Miller WL, Crabtree BF. Investigación clínica. In: Denzin NK, Lincoln YS, coordinadores. Manual de Investigación Cualitativa Vol. III. Las estrategias de investigación cualitativa. Barcelona: Editorial Gedisa; 2013. p. 440-504.

2. Jiménez ME. Desafíos de la investigación cualitativa en salud. Gac Med Bol 2011; 34(2):105-106.

3. Lincoln IS. Sympathetic Connections between Qualitative Methods and Health Research. Qualitative Health Research 1992; 2(4):375-391.

4. Arias MM, Giraldo CV. El rigor científico en la investigación cualitativa. Invest Educ Enferm 2011; 29(3):500-514.

5. Martínez-Salgado C. El muestreo en investigación cualitativa. Principios básicos y algunas controversias. Cien Saude Colet 2012; 17(3):613-619.

6. Cornejo M. Rigor y calidad metodológicos: un reto a la investigación social cualitativa. Psicoperspectivas 2011; 10(2):12-34.

7. Oliva P, Buhring K. Investigación cualitativa y evidencia en salud: Respuestas fundamentales para su comprensión. Rev Chil Salud Pública 2011; 15(3):173-179. 